\title{
Erratum to: Large-Amplitude Periodic Solutions for Differential Equations with Delayed Monotone Positive Feedback
}

\author{
Tibor Krisztin • Gabriella Vas
}

Published online: 28 March 2014

C) Springer Science+Business Media New York 2014

\section{Erratum to: J Dyn Diff Equat (2011) 23:727-790 DOI 10.1007/s10884-011-9225-2}

The purpose of this note is to correct a mistake left in our previous paper [2]. The paper concerns the scalar equation

$$
\dot{x}(t)=-\mu x(t)+f(x(t-1))
$$

with $\mu=1$ and a special strictly increasing, continuously differentiable $f$. The natural phase space for Eq. (1) is $C=C([-1,0], \mathbb{R})$ equipped with the supremum norm. For any $\varphi \in C$, there is a unique solution $x^{\varphi}:[-1, \infty) \rightarrow \mathbb{R}$ of (1). For each $t \geq 0$, the segment $x_{t}^{\varphi} \in C$ is defined by $x_{t}^{\varphi}(s)=x^{\varphi}(t+s),-1 \leq s \leq 0$. Let $\Phi$ denote the semiflow induced by Eq. (1):

$$
\Phi:[-1, \infty) \times C \ni(t, \varphi) \mapsto x_{t}^{\varphi} \in C
$$

Theorem 1.1 of paper [2] gives a periodic solution $p: \mathbb{R} \rightarrow \mathbb{R}$ of Eq. (1) with $p(-1)=0$ and $\dot{p}(-1) \neq 0$. The proof of Theorem 1.2 in Section 8 then applies a Poincaré return map defined on a neighborhood of $p_{0}$ in $H$, where $H=\{\varphi: \varphi(-1)=0\}$ is a hyperplane transversal to the periodic orbit $\mathcal{O}_{p}=\left\{p_{t}: t \in \mathbb{R}\right\}$. As we shall see, this hyperplane was not selected appropriately.

We evoke results from Floquet theory before pointing at the error and showing its correction.

The online version of the original article can be found under doi:10.1007/s10884-011-9225-2.

T. Krisztin $(\bowtie) \cdot$ G.Vas

MTA-SZTE Analysis and Stochastics Research Group, Bolyai Institute,

University of Szeged, Szeged, Hungary

e-mail: krisztin@math.u-szeged.hu

G. Vas

e-mail: vasg@math.u-szeged.hu 


\section{Floquet Theory}

Let $\omega \in(1,2)$ denote the minimal period of $p$. Consider the period map $Q=\Phi(\omega, \cdot)$ with fixed point $p_{0}$. Consider its derivative $M=D_{2} \Phi\left(\omega, p_{0}\right)$ at $p_{0}$. Then $M \varphi=u_{\omega}^{\varphi}$ for all $\varphi \in C$, where $u^{\varphi}:[-1, \infty) \rightarrow \mathbb{R}$ is the solution of the variational equation

$$
\dot{u}(t)=-u(t)+f^{\prime}(p(t-1)) u(t-1)
$$

with $u_{0}^{\varphi}=\varphi$. $M$ is called the monodromy operator. $M$ is a compact operator, 0 belongs to its spectrum $\sigma=\sigma(M)$, and eigenvalues of finite multiplicity-the so called Floquet multipliers-form $\sigma(M) \backslash\{0\}$.

As $\dot{p}$ is a nonzero solution of the variational equation, 1 is a Floquet multiplier with eigenfunction $\dot{p}_{0}$. The paper [2] proves that $\mathcal{O}_{p}$ is hyperbolic, which means that the generalized eigenspace of $M$ corresponding to the eigenvalue 1 is one-dimensional, furthermore there are no Floquet multipliers on the unit circle besides 1 .

If $\varphi$ is a nonzero element of the phase space $C=C([-1,0], \mathbb{R})$, let $V(\varphi)$ denote the number of sign changes of $\varphi$ if it is even or $\infty$, otherwise let $V(\varphi)$ be the number of sign changes plus one. This is the so-called discrete Lyapunov functional of Mallet-Paret and Sell [4].

By Section 4 of [2], $\mathcal{O}_{p}$ has two real and simple Floquet multipliers $\lambda_{1}$ and $\lambda_{2}$ outside the unit circle with $\lambda_{1}>\lambda_{2}>1$. Regarding the associated eigenspaces, we have the following information from [4] and from Appendix VII of [3]. The eigenvector $u_{1}$ of $M$ corresponding to $\lambda_{1}$ is strictly positive. The realified generalized eigenspace $C_{<\lambda_{1}}$ associated with the spectral set $\left\{z \in \sigma:|z|<\lambda_{1}\right\}$ satisfies

$$
C_{<\lambda_{1}} \cap V^{-1}(0)=\emptyset \text {. }
$$

Let $C_{\leq \rho}, \quad \rho>0$, denote the realified generalized eigenspace of $M$ associated with the spectral set $\{z \in \sigma:|z| \leq \rho\}$. The set

$$
\left\{\rho \in(0, \infty): \sigma(M) \cap \rho S_{\mathbb{C}}^{1} \neq \emptyset, C_{\leq \rho} \cap V^{-1}(\{0,2\})=\emptyset\right\}
$$

is nonempty and has a maximum $r_{M}$. Then

$$
C_{\leq r_{M}} \cap V^{-1}(\{0,2\})=\emptyset, \quad C_{r_{M}<} \backslash\{\hat{0}\} \subset V^{-1}(\{0,2\}) \text { and } \operatorname{dim} C_{r_{M}<} \leq 3,
$$

where $C_{r_{M}<}$ is the realified generalized eigenspace of $M$ associated with the nonempty spectral set $\left\{z \in \sigma:|z|>r_{M}\right\}$. It follows from the construction of $p$ in [2] that $V\left(\dot{p}_{0}\right)=2$. Hence $r_{M}<1$ in our case, and $V\left(u_{2}\right)=2$ for the eigenvector $u_{2}$ of $M$ corresponding to $\lambda_{2}$.

\section{Poincaré Return Maps}

Choose $X$ to be a hyperplane with codimension 1 so that $\dot{p}_{0} \notin X$. An application of the implicit function theorem yields a convex bounded open neighborhood $N$ of $p_{0}$ in $C, \varepsilon \in$ $(0, \omega)$ and a $C^{1}$-map $\gamma: N \rightarrow(\omega-\varepsilon, \omega+\varepsilon)$ with $\gamma\left(p_{0}\right)=\omega$ so that for each $(t, \varphi) \in$ $(\omega-\varepsilon, \omega+\varepsilon) \times N$, the segment $x_{t}^{\varphi}$ belongs to $p_{0}+X$ if and only if $t=\gamma(\varphi)$ (see [1], Appendix I in [3]). The Poincaré return map $P_{X}$ is defined by

$$
P_{X}: N \cap\left(p_{0}+X\right) \ni \varphi \mapsto \Phi(\gamma(\varphi), \varphi) \in p_{0}+X .
$$

Then $P_{X}$ is continuously differentiable with fixed point $p_{0}$. 
Let $\sigma\left(P_{X}\right)$ and $\sigma(M)$ denote the spectra of $D P_{X}\left(p_{0}\right): X \rightarrow X$ and the monodromy operator $M$, respectively. We obtain the following result from Theorem XIV.4.5 in [1].

Lemma (i) $\sigma\left(P_{X}\right) \backslash\{0,1\}=\sigma(M) \backslash\{0,1\}$, and for every $\lambda \in \sigma(M) \backslash\{0,1\}$, the projection along $\mathbb{R} \dot{p}_{0}$ onto $X$ defines an isomorphism from the realified generalized eigenspace of $\lambda$ and $M$ onto the realified generalized eigenspace of $\lambda$ and $D P_{X}\left(p_{0}\right)$.

(ii) $1 \notin \sigma\left(P_{X}\right)$.

In Section 8 of [2] we selected the hyperplane $H=\{\varphi: \varphi(-1)=0\}$ and the associated Poincaré map $P=P_{H}$. It follows from the above proposition that $D P\left(p_{0}\right)$ has exactly two real eigenvalues $\lambda_{1}>\lambda_{2}>1$ outside the unit circle. Let $v_{1}$ and $v_{2}$ denote the eigenvectors of $D P\left(p_{0}\right)$ corresponding to $\lambda_{1}$ and $\lambda_{2}$, respectively. Section 8 of [2] used the statement that $V\left(v_{1}\right)=0$ and $V\left(v_{2}\right)=2$. This is not necessarily true. The mistake can be corrected by selecting a different hyperplane.

Let $C_{s}$ and $C_{u}$ be the closed subspaces of $C$ chosen so that $C=C_{s} \oplus \mathbb{R} \dot{p}_{0} \oplus C_{u}, C_{s}$ and $C_{u}$ are invariant under $M$, and the spectra $\sigma_{s}(M)$ and $\sigma_{u}(M)$ of the induced maps $C_{s} \ni x \mapsto M x \in C_{s}$ and $C_{u} \ni x \mapsto M x \in C_{u}$ are contained in $\{\mu \in \mathbb{C}:|\mu|<1\}$ and $\{\mu \in \mathbb{C}:|\mu|>1\}$, respectively. As $\mathcal{O}_{p}$ has two real and simple Floquet multipliers $\lambda_{1}$ and $\lambda_{2}$ outside the unit circle with eigenvectors $u_{1}$ and $u_{2}$, we have $C_{u}=\left\{c_{1} u_{1}+c_{2} u_{2}\right\}$.

Set $Y=C_{s} \oplus C_{u}$. Then $Y$ is a hyperplane in $C, \dot{p}_{0} \notin Y$ and $C=Y \oplus \mathbb{R} \dot{p}_{0}$.

The special choice of $Y$ and Lemma imply that $\lambda_{i}$ and $u_{i}$ is an eigenvalue-eigenvector pair of $D P_{Y}\left(p_{0}\right)$ for both $i \in\{1,2\}$. In addition, $C_{s}$ and $C_{u}$ are invariant under $D P_{Y}\left(p_{0}\right)$, and the spectra $\sigma_{s}\left(P_{Y}\right)$ and $\sigma_{u}\left(P_{Y}\right)$ of the induced maps $C_{s} \ni x \mapsto D P_{Y}\left(p_{0}\right) x \in C_{s}$ and $C_{u} \ni x \mapsto D P_{Y}\left(p_{0}\right) x \in C_{u}$ are contained in $\{\mu \in \mathbb{C}:|\mu|<1\}$ and $\{\mu \in \mathbb{C}:|\mu|>1\}$, respectively. Summing up, $D P_{Y}\left(p_{0}\right)$ has exactly two real and simple eigenvalues $\lambda_{1}>$ $\lambda_{2}>1$ outside the unit circle, and for the corresponding eigenvectors $u_{1}$ and $u_{2}$, we have the desired properties $V\left(u_{1}\right)=0$ and $V\left(u_{2}\right)=2$.

In accordance, $H$ and $P=P_{H}$ should be changed to $Y$ and $P_{Y}$ in Section 8 of [2]. Then the proof of Theorem 1.2. (found in Section 8 of [2]) becomes correct without any further change.

\section{References}

1. Diekmann, O., van Gils, S.A., Verduyn Lunel, S.M., Walther, H.-O.: Delay equations. Functional, complex, and nonlinear analysis. Springer-Verlag, New York (1995)

2. Krisztin, T., Vas, G.: Large-amplitude periodic solutions for differential equations with delayed monotone positive feedback. J. Dynam. Differential Equations 23(4), 727-790 (2011)

3. Krisztin, T., Walter, H.-O., Wu, J.: Shape, smoothness and invariant stratification of an attracting set for delayed monotone positive feedback. Amer. Math. Soc, Providence, RI (1999)

4. Mallet-Paret, J., Sell, G.R.: Systems of differential delay equations: Floquet multipliers and discrete Lyapunov Functions. J. Differential Equations 125, 385-440 (1996) 